\title{
Instantons in the Langevin dynamics: an application to spin glasses.
}

\author{
A. V. Lopatin, L. B. Ioffe
}

August 12, 2018

\begin{abstract}
We develop a general technique to calculate the probability of transitions over the barriers in spin-glasses in the framework of the dynamical theory. We use Lagrangian formulation of the instanton dynamics in which the transitions are represented by instantons. We derive the full set of the equations that determine the instantons but instead of solving them directly we prove that an instanton process can be mapped into a usual process going back in time which simplifies the problem significantly. We apply this general considerations to a simple example of the spherical Sherrington-Kirkpatrick model and we find the probability of the transition between the metastable states which is in agreement with physical expectations.
\end{abstract}

\section{Introduction}

It is well known that all spin-glass systems have complicated free energy structures with many metastable states separated by large barriers [1]. The calculation of heights of these barriers is a difficult task even for the longrange spin-glass models because most of the methods developed in the spin glass theory do not give such information. For example, the application of the standard replica method [1] allows to find the equilibrium free energy which does not contain any information about the heights of the barriers. The standard dynamical approach [6, 3] gives the properties of different metastable states and their history dependence and might contain, in principle, the information about the transition rates between them but in the limit of long-range 
interaction the probability of transitions becomes exponentially low in the number of spins, $N$, and, thus, such processes are neglected by the mean field derivation of the equations for the correlation and response functions on which this aproach is usually based. The modification of the replica method that allows to estimate the energy barriers between metastable states was suggested in Ref.[2]. The main idea of this modification is to study the free energy of the state constrained to have a certain overlap with the given state. The main drawback of it is that it is not clear whether the state corresponding to the energy maximum found by this method is dynamically accessible and that it is indeed a bottleneck of a transition process. The problem is exacerbated by the fact that the replica method weights all states with the Boltzmann weight so it might miss the rare saddle points of low energy in favour of more abundant high energy ones. In this paper we develop an alternative technique which is based on the modified dynamical approach 88 for the calculation of the barriers between the metastable states.

Before we discuss how to modify the dynamical theory so it does not neglect rare processes such as transitions over the barriers we briefly review the standard dynamical approach to the spin-glasses. In this approach one starts from the Lagrangian formulation of the Langevin dynamics, averages over the disorder and, making the saddle point approximation, arrives at a closed system of equations for the sigle site spin-spin correlation function $D\left(t_{1}, t_{2}\right)$ and response function $G\left(t_{1}, t_{2}\right)$. The corrections to the saddle point approximation are small in $1 / N$ because interaction has a range $N$. For example, in the case of the spherical p-spin interacting model one gets a set of integro-differential equations which were solved numerically (and partially analytically); the solution is made possible by the fact that these equations are forward propagating in time which is in turn due to the causality of the Langevin equations and initial conditions imposed in the past [3, 4. By construction these mean field equations describe the most probable evolution of the system in time and ignore all rare processes such as transitions over the barriers. Empirically we visualize the processes that give the main contribution to the conventional dynamical theory as motion down the energy landscape or small fluctuations near the bottom of the valley.

Consider now a typical dynamical process that corresponds to the transition between two close metastable states in a free energy landscape shown in Fig. 1. The system initially is in the state 1 and we want to find the probability of the transition to the state 2 . The path from point 1 to point 2 consists of the uphill motion from state 1 to the unstable stationary point 3 
and the motion from unstable point 3 to stable state 2. Only the first part of the motion corresponds to the rare process, and, therefore, the probability of the transition between the states 1 and 2 is determined by the uphill motion from 1 to 3 . In the Lagrangian formulation of the Langevin dynamics the uphill motion can be described as an instanton which action gives the probability of the process. Note that to find this solution one needs to "force" the system to go upward, i.e. one needs to apply a boundary condition in future (at point 3 in our example) that destroys causality of the theory. This complicates enormously the dynamical equations describing the instanton motion compared to the dynamical equations describing typical processes (such as motion from 3 to 2 ).

The main result of this paper is that an instanton motion can be mapped into a usual motion going back in time. In our example it means that the uphill motion from point 1 to point 3 can be mapped into the usual downhill motion from point 3 to point 1 . This allows one to solve the usual forward propagating equations instead of solving the complicated equations describing the instanton motion. In general, this method does not allow one to find the barrier beween one given state and another but it allows one to find some instanton processes which, hopefully, correspond to typical barriers in a system. In the simple example of a spherical Sherrington-Kirkpatrick model which we consider here it gives the barrier that separates doubly degenerate ground states that differ by the sign of the magnetization; this barrier is physically important because it controls the decay of the ground state magnetization in this system. In this model there are only two locally stable states and $N$ locally unstable ones, naturally one expects that these unstable states are saddle points and the trajectory which connects two ground states must go through one of them but these general qualitative arguments do not indicate which of these saddle points should be used. Qualitatively the problem is that even in this simple model the lowest saddle point might not connect two different minima but connect one minima to itself (false pass). Our approach proves that it is sufficient to climb up to the lowest of them in order to get from one ground state to another.

Further, we confirm that in case when the free energy landscape of the problem is known explicitly, the probability of the transition obtained as an action of the instanton solution is $e^{-\Delta F / T}$ where $\Delta F$ is the difference between the free energy at the end of the instanton trajectory (point 3) and the free energy of the stationary state (point 1). The free energy at the unstable fixed point 3 should be understood as $F=E-T S$, where $E$ is the energy and 


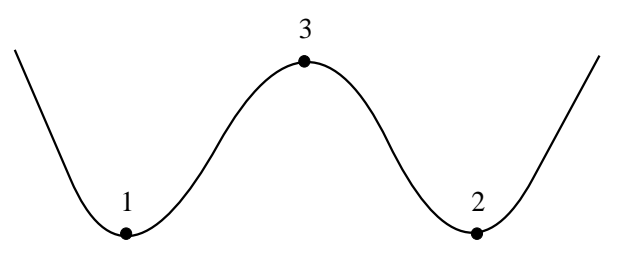

Figure 1: Energy landscape illustrating a transition between states 1 and 2 via saddle point 3 . Uphill motion from 1 to 3 is a rare process which is missed by conventional mean field dynamical equations for the spin glasses, the action corresponding to this process is large in $N$. Downhill motion from 3 to 2 does not cost any action and is described by the conventional dynamic equations, its action is zero.

$S$ is the entropy defined as a logarithm of the configuration space restricted to the direction perpendicular to the trajectory. Of course, there is no much need to calculate the transition probability if the energy of the saddle point in known exactly and it is established that the saddle point is dynamically accessible; the advantage of the method is that it can be also used in the cases where energy lanscape can not be found explicitly, e.g. in all problems in which the disorder average was performed first.

The paper is organized as follows: In Section 2 we prove that an instanton equation of motion can be mapped into a usual equation of motion reversed in time. In Section 3 we consider an instanton transition in the spherical SK model. Section 1 summarizes our results.

\section{Instanton equations}

We start from the Langevin equation describing the overdamped relaxation of the system with energy $\mathcal{H}$

$$
\Gamma_{0}^{-1} \partial_{t} \sigma_{i}=-\frac{\delta \beta \mathcal{H}}{\delta \sigma_{i}}+\xi_{i}
$$

Here $\xi_{i}$ is the Langevin noise with the correlator

$$
\left\langle\xi_{i}\left(t_{1}\right) \xi_{j}\left(t_{2}\right)\right\rangle=2 \Gamma_{0}^{-1} \delta_{i, j} \delta\left(t_{1}-t_{2}\right)
$$

Below we shall choose the time units so that $\Gamma_{0}=1$. Using the standard approach [1] we get a path integral formulation of the problem with the 
Lagrangian

$$
\mathcal{L}=\sum_{i}-\hat{\sigma}_{i}^{2}-i \hat{\sigma}_{i}\left(\partial_{t} \sigma_{i}+\frac{\delta \beta \mathcal{H}}{\delta \sigma_{i}}\right)+\frac{1}{2} \frac{\delta^{2} \beta \mathcal{H}}{\delta \sigma_{i}^{2}} .
$$

The Green functions are defined by

$$
\begin{aligned}
\mathcal{G}_{i, j}\left(t_{1}, t_{2}\right) & \equiv\left[\begin{array}{ll}
\hat{D}_{i, j}\left(t_{1}, t_{2}\right) & G_{i, j}^{\dagger}\left(t_{1}, t_{2}\right) \\
G_{i, j}\left(t_{1}, t_{2}\right) & D_{i, j}\left(t_{1}, t_{2}\right)
\end{array}\right] \\
& =\int D \sigma D \hat{\sigma} e^{\int_{t_{i}}^{t_{f}} \mathcal{L} d t}\left[\begin{array}{c}
i \hat{\sigma}_{i}\left(t_{1}\right) \\
\sigma_{i}\left(t_{1}\right)
\end{array}\right]\left[i \hat{\sigma}_{j}\left(t_{2}\right), \sigma_{j}\left(t_{2}\right)\right],
\end{aligned}
$$

and the dynamical action $A$ is defined by

$$
e^{A}=\int D \sigma D \hat{\sigma} e^{\int_{t_{i}}^{t_{f}} \mathcal{L} d t} .
$$

Usually one fixes only initial boundary conditions. In this case the Green function $\mathrm{G}$ is casual, the anomalous Green function $\hat{D}$ is zero, and the action vanishes. But if one considers rare processes as transitions over the barriers, then one should also fix the final boundary conditions. In that case the Green function $\mathrm{G}$ does not need to be causal, and the action and the Green function $\hat{D}$ do not necessarily vanish.

Now we construct a mapping between an uphill motion (with a negative action monotonically decreasing in time) and the downhill motion (with zero action) going back in time. Consider arbitrary instanton process $(\sigma(t), \widehat{\sigma}(t))$ and impose both initial and final boundary conditions. Applying the transformation

$$
[i \hat{\sigma}, \sigma] \rightarrow\left[i \hat{\sigma}+\vec{\partial}_{t} \sigma, \sigma\right]
$$

to the Lagrangian (2) we get

$$
\begin{aligned}
\mathcal{L} \rightarrow & \mathcal{L}_{n}=\sum_{i}-\hat{\sigma}_{i}^{2}-i \hat{\sigma}_{i}\left(-\partial_{t} \sigma_{i}+\frac{\delta \beta \mathcal{H}}{\delta \sigma_{i}}\right)+\frac{1}{2} \frac{\delta^{2} \beta \mathcal{H}}{\delta \sigma_{i}^{2}} \\
& +\beta\left(H\left(t_{f}\right)-H\left(t_{i}\right)\right)
\end{aligned}
$$

Note that this Lagrangian differs from the original Lagrangian (2) only by the sign of time derivative and constant boundary term. Therefore inverting time in the Lagrangian (6) one can make the Lagrangians (2, 60) equivalent. 
Therefore, the Lagrangian (6) describes normal downhill motion formally inverted in time.

The Green function defined as averaged with respect to Lagrangian (3)

$$
\mathcal{G}\left(t_{1}, t_{2}\right)=\left\langle\left[\begin{array}{c}
i \hat{\sigma}\left(t_{1}\right) \\
\sigma\left(t_{1}\right)
\end{array}\right]\left[i \hat{\sigma}\left(t_{2}\right), \sigma\left(t_{2}\right)\right]\right\rangle_{\mathcal{L}}
$$

and the Green function defined as averaged with respect to the Lagrangian (6)

$$
\mathcal{G}_{n}\left(t_{1}, t_{2}\right)=\left\langle\left[\begin{array}{c}
i \hat{\sigma}\left(t_{1}\right) \\
\sigma\left(t_{1}\right)
\end{array}\right]\left[i \hat{\sigma}\left(t_{2}\right), \sigma\left(t_{2}\right)\right]\right\rangle_{\mathcal{L}_{n}}
$$

are related by

$$
\mathcal{G}\left(t_{1}, t_{2}\right)=\left[\begin{array}{cc}
1 & \vec{\partial}_{t 1} \\
0 & 1
\end{array}\right] \mathcal{G}_{n}\left(t_{1}, t_{2}\right)\left[\begin{array}{cc}
1 & 0 \\
\overleftarrow{\partial}_{t 2} & 1
\end{array}\right]
$$

Because the Green function $\mathcal{G}_{n}$ corresponds to the normal downhill process inverted in time, it should have the form

$$
\mathcal{G}_{n}=\left[\begin{array}{cc}
0 & G_{n}^{\dagger} \\
G_{n} & D_{n}
\end{array}\right]
$$

Therefore the transformation (9) in components is

$$
\begin{gathered}
D\left(t_{1}, t_{2}\right)=D_{n}\left(t_{1}, t_{2}\right) \\
G\left(t_{1}, t_{2}\right)=G_{n}\left(t_{1}, t_{2}\right)+\partial_{2} D_{n}\left(t_{1}, t_{2}\right) \\
\hat{D}\left(t_{1}, t_{2}\right)=\partial_{1} G_{n}\left(t_{1}, t_{2}\right)+\partial_{2} G_{n}^{\dagger}\left(t_{1}, t_{2}\right)+\partial_{1} \partial_{2} D_{n}\left(t_{1}, t_{2}\right) .
\end{gathered}
$$

Note that the response function $G_{n}$ should be purely advanced due to formal inversion of time with respect to the downhill motion.

Thus, in order to construct the Green functions for the instanton processes one should find the Green functions for the corresponding normal process, invert time, and apply the transformation (9).

Now we show that the action can be expressed through the energies and configuration spaces of the initial and final states: Suppose that initially the system is at the stable state $s$, and we want to find the probability to escape this state going trough the unstable fixed point $u$ which is the final state of the instanton motion. Note that in all the above considerations we 


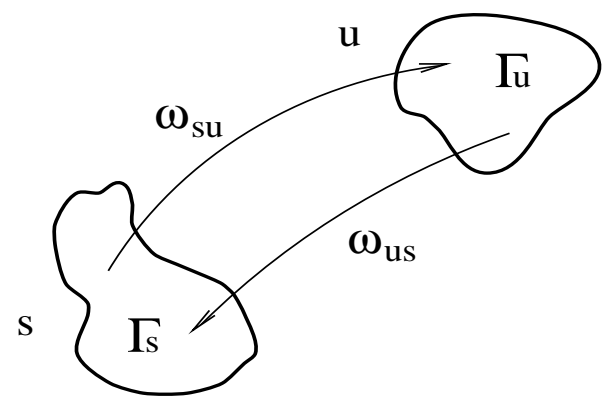

Figure 2:

assumed completely fixed boundary conditions for $\sigma_{i}$. But physically, initial and final states correspond to some regions in configuration states which we denote as $\Gamma_{s}$ and $\Gamma_{u}$ respectively. To emphasize the difference between the process with completely fixed boundary conditions and the processes with physical boundary conditions we refer to the former as elementary processes. According to Eqs.(2) 6) the probability of an elementary process of motion from $s$ to $u\left(w_{s u}\right)$ is related to the elementary process of motion from $u$ to $s$ $\left(w_{u s}\right)$ through

$$
w_{s u}=w_{u s} e^{\beta\left(E\left(t_{f}\right)-E\left(t_{i}\right)\right)} .
$$

The probability to escape the state $s$ is

$$
W_{s u}=\Gamma_{u} w_{s u} .
$$

On the other hand the probability to go from the unstable state $u$ to the stable state $s$ is 1 , therefore

$$
W_{u s}=w_{u s} \Gamma_{s}=1 .
$$

Combining Eqs. 14, 15, 16) for the probability to escape the stable state $u$ we get

$$
W_{s u}=\exp \left[-\beta\left(E\left(t_{f}\right)-E\left(t_{i}\right)\right)+S\left(t_{f}\right)-S\left(t_{i}\right)\right],
$$

where $S=\ln \Gamma$ is the entropy. Note that at the stable point the entropy $S\left(t_{i}\right)$ is just the equilibrium entropy corresponding to this state. The entropy of the final state $S\left(t_{f}\right)$ can not be defined thermodynamically because it corresponds 
to the configuration space of the unstable state. Defining the free energy as $F=E-T S$ one can write (17) as

$$
W_{s u}=e^{-\beta F\left(t_{f}\right)+\beta F\left(t_{i}\right)} .
$$

\section{Instanton transition in the spherical SK model.}

The Hamiltonian of the spherical SK model is

$$
\mathcal{H}=\frac{1}{2} \sum \sigma_{i} J_{i j} \sigma_{j}
$$

with the spherical constraint $\sum_{i} \sigma_{i} \sigma_{i}=N$ imposed. The Hamiltonian becomes diagonal in the basis of eigenvectors of the matrix $J_{i, j}$

$$
\mathcal{H}=\frac{1}{2} \sum_{\mu} \epsilon_{\mu} s_{\mu}^{2}
$$

where

$$
\begin{aligned}
\sum_{j} J_{i, j} \sigma_{j}^{\mu} & =\epsilon_{\mu} \sigma_{i}^{\mu}, \\
\sigma_{i} & =\sum_{\mu} s_{\mu} \sigma_{i}^{\mu} .
\end{aligned}
$$

The Lagrangian corresponding to this model is

$$
\mathcal{L}(s, \lambda)=-\sum_{\mu}\left[\hat{s}_{\mu}^{2}+i \hat{s}_{\mu}\left(\partial_{t}+\epsilon_{\mu}+\lambda\right) s_{\mu}\right]+\frac{1}{2}(N+2) \lambda,
$$

where $\lambda$ is the time-dependent Lagrange multiplier field which appears in the equation of motion due to the constraint and we take $\beta=1$ for convenience. The functional integral over the variables $s_{\mu}, \widehat{s}_{\mu}$ should be performed with the weight that includes $\delta$-function that ensures the constraint. Using the integral representation of this $\delta$-function

$$
\delta\left(\sum_{\mu} s_{\mu}^{2}-N\right)=\int D \phi e^{i \int d t \phi\left(\sum_{\mu} s_{\mu}^{2}-N\right)}
$$

we get the following Lagrangian

$$
\begin{aligned}
\mathcal{L}(s, \hat{s}, \lambda, \phi)= & \sum_{\mu}\left(-\hat{s}_{\mu}^{2}-i \hat{s}_{\mu}\left(\partial_{t}+\epsilon_{\mu}+\lambda\right) s_{\mu}+i \phi s_{\mu}^{2}\right) \\
& +\frac{1}{2}(N+2) \lambda-i \phi N .
\end{aligned}
$$


At low temperatures the condensation into the lowest eigenvalue $\mu=0$ eventually takes place. Therefore we introduce the condensate $S_{0}$ and integrate over $s_{\mu}$ with $\mu \geq 1$ getting

$$
\begin{gathered}
\mathcal{L}=-\hat{S}_{0}^{2}-i \hat{S}_{0}\left(\partial+\epsilon_{0}+\lambda\right) S_{0}+i \phi S_{0}^{2}+\frac{1}{2}(N+2) \lambda-i \phi N \\
-\frac{1}{2} \sum_{\mu \geq 1} \operatorname{Tr} \ln \mathcal{G}_{\mu},
\end{gathered}
$$

where the matrix Green function

$$
\mathcal{G}_{\mu}=\left[\begin{array}{ll}
\hat{D}_{\mu}\left(t_{1}, t_{2}\right) & G_{\mu}^{\dagger}\left(t_{1}, t_{2}\right) \\
G_{\mu}\left(t_{1}, t_{2}\right) & D_{\mu}\left(t_{1}, t_{2}\right)
\end{array}\right]
$$

satisfies the equation

$$
\left[\begin{array}{cc}
-2 & \partial_{t 1}+\lambda\left(t_{1}\right)+\epsilon_{\mu} \\
-\partial_{t 1}+\lambda\left(t_{1}\right)+\epsilon_{\mu} & \phi\left(t_{1}\right)
\end{array}\right] \mathcal{G}_{\mu}=\delta\left(t_{1}-t_{2}\right) .
$$

The number of cites $N$ is large, therefore we will perform the integrals with the weight $\exp \left(\int \mathcal{L} d t\right)$ where $\mathcal{L}$ is given by (26) in the saddle point approximation. Taking the variation with respect to $\phi, \lambda, \hat{S}_{0}, S_{0}$ and transforming the variables via $-2 i \phi \rightarrow \phi, i \hat{S} \rightarrow \sqrt{N} \hat{S}$ we get the saddle point equations

$$
\begin{aligned}
\frac{1}{N} \sum_{\mu \geq 1} D_{\mu}+S_{0}^{2} & =1, \\
\frac{1}{N} \sum_{\mu \geq 1} G_{\mu}+\hat{S}_{0} S_{0} & =0, \\
\left(\partial+\epsilon_{0}+\lambda\right) S_{0}-2 \hat{S}_{0} & =0, \\
\left(-\partial+\epsilon_{0}+\lambda\right) \hat{S}_{0}+\phi S_{0} & =0,
\end{aligned}
$$

where

$$
D_{\mu}(t)=D_{\mu}(t, t), \quad G_{\mu}(t)=G_{\mu}(t, t+\delta) .
$$

Note that Eqs.(29-32) contain only the equal time Green functions. Therefore it is convenient to write the equations directly for the equal time Green functions instead of $(28)$ :

$$
\begin{aligned}
& \partial G_{\mu}=2 \hat{D}_{\mu}+\phi D_{\mu}, \\
& \partial \hat{D}_{\mu}=2\left(\lambda+\epsilon_{\mu}\right) \hat{D}_{\mu}+\phi\left(1+2 G_{\mu}\right), \\
& \partial D_{\mu}=-2\left(\lambda+\epsilon_{\mu}\right) D_{\mu}+2\left(1+2 G_{\mu}\right),
\end{aligned}
$$


We assume that the system is initially at the equilibrium. This corresponds to the stationary solution of Eqs. (29-35)

$$
\begin{array}{r}
\frac{1}{N} \sum_{\mu \geq 1} D_{\mu}+S_{0}^{2}=1, \\
D_{\mu}=\frac{1}{\lambda_{s}+\epsilon_{\mu}}, \\
\lambda_{s}=-\epsilon_{0}, \\
G_{\mu}=\hat{D}_{\mu}=\hat{S}_{0}=\phi=0,
\end{array}
$$

where $\lambda_{s}$ is the value of $\lambda$ for this stable stationary solution. Eq.(38) follows from Eq.(31) because we assumed that there is a nonzero condensate density $S_{0}$. Note that there are two equilibrium states with $S_{0}>0$ and $S_{0}<0$.

Our goal is to find the probability of the instanton transition from one state to the other. For definiteness, assume that the initial state is one with $S_{0}>0$. Obviously, $S_{0}$ first decreases to zero during the instanton process and then it becomes negative. Only the first uphill part of the motion gives the contribution to the action, therefore we will consider only this uphill part of the trajectory. The end point of this uphill motion corresponds to an unstable fixed point of Eqs. 229 35). The condensate density $S_{0}$ is zero at this point therefore from Eqs.(29-35) we get the following equations corresponding to this stationary (but unstable) solution:

$$
\begin{aligned}
\frac{1}{N} \sum_{\mu \geq 1} D_{\mu} & =1 \\
D_{\mu} & =\frac{1}{\lambda_{u}+\epsilon_{\mu}}, \\
G_{\mu} & =\hat{D}_{\mu}=\hat{S}_{0}=\phi=0,
\end{aligned}
$$

where $\lambda_{u}$ is the value of $\lambda$ at this unstable stationary solution. Physically, it is natural to expect that that this solution corresponds to the condensate in the first eigenstate $\mu=1$. Indeed, taking $\lambda_{u}=-\epsilon_{1}+\frac{1}{S_{1}^{2}}$, where $S_{1}$ is the condensate at the first eigenstate $\mu=1$, we get

$$
\begin{aligned}
\frac{1}{N} \sum_{\mu \geq 2} D_{\mu}+S_{1}^{2} & =1 \\
D_{\mu} & =\frac{1}{\lambda_{u}+\epsilon_{\mu}} .
\end{aligned}
$$


These equations are similar to Eqs.(36-38) for the stable fixed point with the difference that the system condenses into the first eigenstate.

Now we need to find the trajectory connecting the fixed points mentioned above. It was shown in the previous section that an uphill trajectory can be mapped into a downhill trajectory going back in time. To show this, according to Eqs.(5, 12), one should take

$$
\begin{aligned}
G_{\mu} & =\frac{1}{2} \partial D_{\mu}, \\
\hat{S}_{0} & =\partial S_{0},
\end{aligned}
$$

where $D_{\mu}, S_{0}$ should satisfy the downhill equations with inverse time

$$
\begin{aligned}
\frac{1}{N} \sum_{\mu \geq 1} D_{\mu}+S_{0}^{2} & =1 \\
\left(-\partial+\epsilon_{0}+\lambda\right) S_{0} & =0, \\
-\partial D_{\mu} & =-2\left(\lambda+\epsilon_{\mu}\right) D_{\mu}+2
\end{aligned}
$$

Indeed, taking

$$
\begin{aligned}
\phi & =\partial \lambda \\
\hat{D}_{\mu} & =\frac{1}{4} \partial^{2} D_{\mu}-\frac{1}{2} D_{\mu} \partial \lambda
\end{aligned}
$$

along with Eqs.(45,46), one can show that Eqs.(29-35) are reduced to Eqs.(4749). The trajectory connecting the stable and unstable saddle points can be found analytically; (see Appendix A) the result is

$$
\begin{aligned}
\lambda(t)+\epsilon_{0}= & \left(\lambda_{u}+\epsilon_{0}\right) f\left[2\left|\lambda_{u}+\epsilon_{0}\right|\left(t_{0}-t\right)\right] \\
D_{\mu}(t)= & \frac{1}{\lambda_{u}+\epsilon_{\mu}} f\left[2\left|\lambda_{u}+\epsilon_{0}\right|\left(t_{0}-t\right)\right] \\
& +\frac{1}{\epsilon_{\mu}} f\left[2\left|\lambda_{u}+\epsilon_{0}\right|\left(t-t_{0}\right)\right],
\end{aligned}
$$

where

$$
f[x]=\frac{1}{e^{x}+1},
$$

where $t_{0}$ is an arbitrary finite time which reflects the translational invariance in time. 
The last step is to calculate the action which determines the probability of the instanton process. Note, that to find it, one should take $\operatorname{Tr} \ln$ of the operator containing $\lambda, \phi$ which are functions of time. The necessary calculation is presented in Appendix B, and the answer, simplified with the help of Eqs.(29.35), is

$$
A / N=\frac{1}{2} \int d t\left(\phi+\frac{2}{N} \sum_{\mu \geq 1} \frac{G_{\mu}}{D_{\mu}}\right) .
$$

Using that $\phi=\partial \lambda$ and $G_{\mu}=\frac{1}{2} \partial D_{\mu}$ we get

$$
A / N=\frac{1}{2}\left(\lambda+\frac{1}{N} \sum_{\mu \geq 1} \ln D_{\mu}\right)_{t=t_{i}}^{t=t_{f}} .
$$

Note that at the fixed points $D_{\mu}=1 /\left(\epsilon_{\mu}+\lambda\right)$, therefore one can write

$$
A=-\left[F\left(\lambda\left(t_{f}\right)\right)-F\left(\lambda\left(t_{i}\right)\right)\right]
$$

where $F(\lambda)$ is the equilibrium free energy of this model

$$
F(\lambda) / N=-\lambda / 2+\frac{1}{2 N} \sum_{\mu \geq 1} \ln \left(\epsilon_{\mu}+\lambda\right) .
$$

The result (57) is in the agreement with the general result (18). As we mentioned in Sec.2 the free energy at the unstable fixed point cannot be defined thermodynamically. But in this simple model one can formally eliminate the unstable direction (i.e. impose the constraint $S_{0}=0$ ) and then it becomes possible to define the free energy thermodynamically. That is why we got the difference of the equilibrium free energies in (58). Note that the Lagrange multiplier $\lambda$ changes during the instanton process on $\lambda\left(t_{f}\right)-\lambda\left(t_{i}\right)=\epsilon_{1}-\epsilon_{0}$. The typical distance between neighboring energy levels at the edges of energy level distribution is of order of $1 / \sqrt{N}$. Therefore one can expand (58) in $\Delta \lambda$ getting

$$
A=-\frac{N}{2 T} S_{0}^{2}\left(\epsilon_{1}-\epsilon_{0}\right)
$$

where we restored the temperature $T$. Note that this action is of the order of $\sqrt{N}$. 


\section{Discussion and conclusions.}

We developed the method that simplifies the calculation of the probablity of rare processes such as transitions over the barriers. Our method is based on the Lagrangian approach to the dynamics in which rare processes correspond to the instantons. Generally, in order to obtain the probability of a particular transition between two given states one need to apply a boundary condition in future and in the past, this destroys the causality of the theory: the response function $G=\langle\hat{s} s\rangle$ becomes non causal and the anomalous correlation function $\hat{D}=\langle\hat{s} \hat{s}\rangle$ appears. This complicates the description of the instanton processes.

The main result of this paper is that an instanton process can be mapped into a usual process going back in time. So, knowing the correlation functions of the corresponding normal process one can construct the instanton correlation functions. We showed that this mapping gives the sensible probability to escape a free energy well, $e^{-\Delta F / T}$, where $\Delta F$ is the depth of the free energy well. The free energy at the end of the instanton trajectory cannot be defined thermodynamically because at this point the system is at the unstable equilibrium, instead it should be defined by $F=E-T S$, where $E$ is the energy and $S$ is the statistical entropy at the end of the instanton trajectory, i.e. entropy constrained to the states orthogonal to the descending direction.

We applied this approach to the spherical SK model which usual dynamical properties were studied in Ref. [7]. This model has just two ground states, corresponding to the energy level $\epsilon_{0}$, and no other metastable states. Although the relaxation towards each of these two states is exponential, model exhibits aging behavior when the system relaxes from a random spin configuration to the equilibrium. We considered the instanton transition from one ground state to the other. In accordance with our general result the equations describing the instanton process in this model can be transformed into the usual equations with inverted time. This transformation allows one to find analytically the instanton trajectory. The probability of this transition was found to be $e^{-S_{0}^{2} N\left(\epsilon_{1}-\epsilon_{0}\right) / 2 T}$ where $\epsilon_{1}$ is the energy of the first (unstable) level. It shows that although the system has $N$ saddle points and a complicated phase space the path connecting two ground states with opposite magnetization might go via the saddle point with the lowest energy. The typical distance between the neighboring energy levels at the edge of the en-

ergy spectrum is of order $1 / \sqrt{N}$, therefore the action is of order $\sqrt{N}$. Note that the distance between the energy levels $\epsilon_{1}-\epsilon_{0}$ is different for different 
samples, therefore the transition probability is not a self-averaging quantity. Therefore in this problem it would be very difficult to get the correct answer for the transition probability in any technique which involves averaging at the beginning of calculations.

We hope that this method can be used to find the barriers between the metastable states in more complicated spin glasses like $p>2$ spin models or SK model. The first attempt of application of the instanton method to SK model was done in Ref.[B] It is a more complicated problem because there are many metastable states in these glasses and therefore the averaging should be done at the beginning of calculations. The scaling of the action $\sqrt{N}$ which we got for $p=2$ model is probably specific for the spherical model because the barriers in spin glasses with exponential number of states are due to the nonlinearity of the dynamical equations which is absent in $p=2$ spherical model.

\section{Appendix A}

In this Appendix we will find the trajectory connecting the unstable fixed point with the stable one. It is natural to invert time in Eqs.(47.49) so that they will describe the usual downhill motion:

$$
\begin{aligned}
\frac{1}{N} \sum_{\mu} \tilde{D}_{\mu}+\tilde{S}_{0}^{2} & =1 \\
\left(\partial+\epsilon_{0}+\tilde{\lambda}\right) \tilde{S}_{0} & =0 \\
\partial \tilde{D}_{\mu} & =-2\left(\tilde{\lambda}+\epsilon_{\mu}\right) \tilde{D}_{\mu}+2,
\end{aligned}
$$

where tilde means invertion of time with respect to the instanton motion, for example $\tilde{S}_{0}(t)=S_{0}(-t)$. We need to find the solution of these equatiosn corresponding to the downhill trajectory that begins from the unstable fixed

point and ends at the stable one. Therefore the initial boundary condition is $(t=-\infty)$

$$
\begin{aligned}
\tilde{D}_{\mu}(-\infty) & =\frac{1}{\epsilon_{\mu}+\lambda_{u}}, \quad \mu \geq 1 \\
S_{0} & =0 \\
\tilde{\lambda}(-\infty) & =-\epsilon_{1}+\frac{1}{S_{1}^{2}} \\
\tilde{\lambda}(-\infty) & =\lambda_{u}
\end{aligned}
$$


and the final one $(t=\infty)$ is

$$
\begin{aligned}
\tilde{D}_{\mu}(\infty) & =\frac{1}{\epsilon_{\mu}-\epsilon_{0}}, \quad \mu \geq 1, \\
\tilde{\lambda}(\infty) & =-\epsilon_{0}, \\
S_{0} & \neq 0 .
\end{aligned}
$$

The solution of the Eq.(62) satisfying to the boundary condition (63) is

$$
\tilde{D}_{\mu}(t)=2 \int_{-\infty}^{t} d t^{\prime} e^{-2 \int_{t^{\prime}}^{t}\left[\tilde{\lambda}\left(t^{\prime \prime}\right)+\epsilon_{\mu}\right] d t^{\prime \prime}}
$$

Using Eqs.(61,62) one can write Eq.(60) in the form

$$
\tilde{\lambda}+\epsilon_{0}=-\frac{1}{N} \sum_{\mu}\left(\epsilon_{\mu}-\epsilon_{0}\right) D_{\mu}+1,
$$

which will be more convenient for us. For simplisity let us take $\epsilon_{0}=0$ further in this Appendix. Substitution of Eq.(70) into Eq.(71) gives

$$
\tilde{\lambda}(t)=\frac{2}{N} \sum_{\mu} \int_{-\infty}^{t} d t^{\prime} e^{-2 \int_{t^{\prime}}^{t}\left[\tilde{\lambda}\left(t^{\prime \prime}\right)+\epsilon_{\mu}\right] d t^{\prime \prime}} \tilde{\lambda}\left(t^{\prime}\right) .
$$

Introducing the function

$$
F(t)=\Gamma(t) \tilde{\lambda}(t),
$$

where $\Gamma(t)$ is defined by (up to the multiplication by a constant)

$$
\frac{\Gamma(t)}{\Gamma\left(t^{\prime}\right)}=e^{2 \int_{t^{\prime}}^{t} \tilde{\lambda}\left(t^{\prime \prime}\right) d t^{\prime \prime}},
$$

one can write Eq.(71) as a linear integral equation on $F(t)$

$$
F(t)=\frac{2}{N} \int_{-\infty}^{t} d t^{\prime} F\left(t^{\prime}\right) \sum_{\mu} e^{-2 \epsilon_{\mu}\left(t-t^{\prime}\right)},
$$

which can be easily solved giving

$$
F(t)=A e^{2 \lambda_{u} t},
$$

where $A$ is an arbitrary constant. Now using Eq.(73) one can write

$$
e^{2 \lambda_{u}\left(t-t^{\prime}\right)}=\frac{\lambda(t)}{\lambda\left(t^{\prime}\right)} e^{2 \int_{t^{\prime}}^{t} \tilde{\lambda}\left(t^{\prime \prime}\right) d t^{\prime \prime}},
$$


then taking a logarithm and differentiating with respect to $t$ we get a differential equation on $\tilde{\lambda}$

$$
\partial_{t} \tilde{\lambda}(t)=2 \tilde{\lambda}(t)\left[\tilde{\lambda}_{u}-\tilde{\lambda}(t)\right]
$$

which can be solved giving

$$
\tilde{\lambda}(t)=\tilde{\lambda}_{u} f\left[2\left|\tilde{\lambda}_{u}\right|\left(t-t_{0}\right)\right]
$$

where

$$
f[x]=\frac{1}{e^{x}+1},
$$

and $t_{0}$ is an arbitrary time which reflects the translational invariance in time. In case $\epsilon_{0} \neq 0$ one can easily generalize Eq.(79) to

$$
\tilde{\lambda}(t)+\epsilon_{0}=\left(\tilde{\lambda}_{u}+\epsilon_{0}\right) f\left[2\left|\tilde{\lambda}_{u}+\epsilon_{0}\right|\left(t-t_{0}\right)\right] .
$$

Knowing $\tilde{\lambda}(t)$ one can find $\tilde{D}_{\mu}$

$$
\begin{aligned}
\tilde{D}_{\mu}(t)= & \frac{1}{\lambda_{u}+\epsilon_{\mu}} f\left[2\left|\lambda_{u}+\epsilon_{0}\right|\left(t-t_{0}\right)\right] \\
& +\frac{1}{\epsilon_{\mu}} f\left[2\left|\lambda_{u}+\epsilon_{0}\right|\left(t_{0}-t\right)\right] .
\end{aligned}
$$

\section{Appendix B}

The main problem in calculation of the action is to find

$$
A_{\mu} \equiv \frac{1}{2} \operatorname{Tr} \ln \left[\begin{array}{cc}
-2 & \partial+\lambda+\epsilon_{\mu} \\
-\partial+\lambda+\epsilon_{\mu} & \phi
\end{array}\right] .
$$

Note that taking the variational derivative of (83) with respect to $\lambda(t)$ and $\phi(t)$ we get respectively $G_{\mu}(t, t)=1 / 2+G_{\mu}(t)$ and $\frac{1}{2} D_{\mu}(t)$. The idea of our method of calculation of (83) is to find a functional which gives the same functions $\left(G_{\mu}+\frac{1}{2}\right.$ and $\left.\frac{1}{2} D_{\mu}\right)$ when one takes the variations with respect to $\lambda$ and $\phi$. Up to boundary terms this functional should be equal to the action, and these boundary terms can be found from the requirement that the action should be zero for any downhill trajectory.

Now let us find the functional mentioned above: Eqs.(33-35) have the following invariant

$$
D_{\mu} \hat{D}_{\mu}-\left(1+G_{\mu}\right) G_{\mu}=c
$$


where $c$ is an arbitrary constant. But initially $G_{\mu}=\hat{D}_{\mu}=0$, therefore $c=0$. The condition (84) can be satisfied automatically by introducing the new variables

$$
D_{\mu}=\eta_{\mu} \eta_{\mu}, \quad G_{\mu}=\eta_{\mu} \hat{\eta}_{\mu}-1 / 2, \quad \hat{D}_{\mu}=\hat{\eta}_{\mu} \hat{\eta}_{\mu}-\frac{1}{4 \eta_{\mu}^{2}} .
$$

The new variables $\eta_{\mu}, \hat{\eta}_{\mu}$ satisfy the following equations

$$
\begin{array}{r}
\partial \eta_{\mu}=-\left(\lambda+\epsilon_{\mu}\right) \eta_{\mu}+2 \hat{\eta}_{\mu}, \\
\partial \hat{\eta}_{\mu}=\left(\lambda+\epsilon_{\mu}\right) \hat{\eta}_{\mu}+\phi \eta_{\mu}-\frac{1}{2 \eta_{\mu}^{3}} .
\end{array}
$$

These equations can be obtained by taking the variations of the functional

$$
\Gamma_{\mu}=-\left[\hat{\eta}_{\mu}^{2}-\hat{\eta}_{\mu}\left(\partial+\epsilon_{\mu}+\lambda\right) \eta_{\mu}-\frac{1}{2} \phi \eta_{\mu}^{2}-\frac{1}{4 \eta_{\mu}^{2}}\right]
$$

with respect to $\hat{\eta}$ and $\eta$. Note that the variational derivatives of (83) and $\Gamma_{\mu}$ with respect to $\lambda, \phi$ are the same if we take $\Gamma_{\mu}$ at the saddle point with respect to $\hat{\eta}, \eta$, i.e. Eqs. 86, 87) should be satisfied. Therefore the action $A_{\mu}$ can be written as

$$
A_{\mu}=\Gamma_{\mu}+\left.T\left(\hat{D}_{\mu}(t), D_{\mu}(t), G_{\mu}(t), \lambda(t), \phi(t)\right)\right|_{t=t_{i}} ^{t=t_{f}}
$$

where $T$ is an unknown function. The initial and final times $t_{i}$ and $t_{f}$ correspond to the fixed points of Eqs. 29-35), therefore the abnormal functions $\hat{D}_{\mu}, G_{\mu}, \phi$ should be zero at these points and we can simplify (89)

$$
A_{\mu}=\Gamma_{\mu}+\left.T\left(D_{\mu}(t), \lambda(t)\right)\right|_{t=t_{i}} ^{t=t_{f}} .
$$

Using the saddle point equations (29,35) and Eqs. (86, 87) one can simplify the total action $A$ getting

$$
\begin{array}{r}
A / N=\frac{1}{2} \int d t\left(\phi+\frac{1}{N} \sum_{\mu} \frac{2 G_{\mu}}{D_{\mu}}-\frac{1}{2 N} \sum_{\mu} \partial \ln D_{\mu}\right) \\
+\left.\sum_{\mu} T\left(D_{\mu}(t), \lambda(t)\right)\right|_{t=t_{i}} ^{t=t_{f}}
\end{array}
$$

But this action should be zero for any downhill trajectory, therefore the third and forth terms in (91) should cancel each other, and we finally get

$$
A / N=\frac{1}{2} \int d t\left(\phi+\frac{1}{N} \sum_{\mu} \frac{2 G_{\mu}}{D_{\mu}}\right) .
$$




\section{References}

[1] (see for example) K.H. Fischer and J.A. Hertz, Spin Glasses (Cambridge University Press, 1993)

[2] A.Cavagna, I. Giardina, G. Parisi, cond-mat/9702069

[3] L.F. Cugliandolo and J. Kurchan, Phys. Rev. Let. B 71, 173 (1993)

[4] A. Crisanti, H. Horner, H.-J. Sommers, Z. Phys. B 92, 257 (1993)

[5] T. R. Kirkpatrick, D. Thirumalai, Phys. Rev. B 36 , 5388 (1987)

[6] V. Dotsenko, M. Feigelman and L. B. Ioffe, Spin Glasses and related problems, Soviet Physics Reviews A15, 1-250 (1990); L. B. Ioffe, Phys. Rev. B 38, 5181 (1988).

[7] Leticia F. Cugliandolo and David S. Dean, J. Phys. A 28, 4213 (1995)

[8] L.B. Ioffe, D. Sherrington, Phys. Rev. Rev. B 57, 7666 (1998) 